Supporting Information for:

\title{
Thermodynamic Analysis of Li-Intercalated Graphite by First-Principles Calculations with Vibrational and Configurational Contributions
}

Jun Haruyama, ${ }^{*}{ }^{\dagger}$ Shigeharu Takagi, ${ }^{\dagger}$ Keiji Shimoda, ${ }^{\ddagger}$ Iwao Watanabe, ${ }^{\ddagger}$ Keitaro Sodeyama, ${ }^{\S}$ Tamio Ikeshoji," and Minoru Otani ${ }^{\perp}$

'Institute for Solid State Physics, The University of Tokyo, 5-1-5 Kashiwanoha, Kashiwa, Chiba 277-8581, Japan

Office of Society-Academia Collaboration for Innovation, Kyoto University, Gokasho, Uji, Kyoto 611-0011, Japan

${ }^{\S}$ Research and Services Division of Materials Data and Integrated System (MaDIS), National Institute for Materials Science (NIMS), 1-1 Namiki, Tsukuba, Ibaraki 305-0044, Japan

"Mathematics for Advanced Materials Open Innovation Laboratory (MathAM-OIL), National Institute of Advanced Industrial Science and Technology (AIST), c/o Advanced Institute of Material Research (AIMR), Tohoku University, Sendai 980-8577, Japan ${ }^{\perp}$ Center for Computational Sciences, University of Tsukuba, 1-1-1 Ten-nodai, Tsukuba, Ibaraki 305-8577, Japan

\section{Corresponding Author}

*haruyama@issp.u-tokyo.ac.jp 


\section{S1. Computational details of the $\mathrm{Li}_{x} \mathrm{C}_{6}$ structures and DFT calculations}

The interlayer structures of the $\mathrm{AB}$ and mixed stacks were constructed as follows. An odd stage of the $\mathrm{AB}$ stack forms a double super-periodic C layer structure, as shown in Fig. S1(a). Conversely, in an even stage, the $\mathrm{Li}$-stacking structure in the $\mathrm{AB}$ stack is single periodic for each layer regardless of its stage number. The even/odd stages of the mixed stack form double-super-periodic/single-periodic structures, as shown in Fig. S1(b). The Li-stacking structures in the mixed stack are also double-super-periodic/single-periodic structures for even/odd stages. Furthermore, the double-periodic Li-stacking structures of $\mathrm{Li} / 9 \mathrm{C}, \mathrm{Li} / 12 \mathrm{C}$, $\mathrm{Li} / 18 \mathrm{C}, \mathrm{Li} / 24 \mathrm{C}$, and $\mathrm{Li} / 36 \mathrm{C}$ were arranged, as shown in Fig. S1(c). Finally, the crystal structures of AA-C, AB-C, and bcc Li are shown in Fig. S1(d).

Quantum ESPRESSO (QE) was used to perform spin-unpolarized DFT calculations with a plane-wave basis. $^{\mathrm{S} 1, \mathrm{~S} 2}$ The electronic configurations used in the ultrasoft pseudopotentials ${ }^{\mathrm{S} 3-\mathrm{S} 6}$ were $1 \mathrm{~s}^{2} 2 \mathrm{~s}^{1}$ for Li and $2 s^{2} 2 p^{2}$ for $\mathrm{C}$ with nonlinear core correction. ${ }^{\mathrm{S} 7}$ The DFT calculations were performed with the vdW-DF2B86 $\mathrm{R}^{\mathrm{S} 8}$ exchange-correlation functional for the enthalpy terms. The cutoff energies were set to 40/320 Ry and 80/640 Ry $(1 \mathrm{Ry}=13.606 \mathrm{eV})$ for the wave functions/augmented charge of $\mathrm{Li}_{x} \mathrm{C}_{6}$ and bcc $\mathrm{Li}$, respectively. Shifted k-point sampling of $24 \times 24,16 \times 16,8 \times 8,8 \times 8$, and $4 \times 4$ grids was used for the in-plane structures of $\mathrm{C}, \mathrm{Li} / 6 \mathrm{C}, \mathrm{Li} / 9 \mathrm{C}$ (and $\mathrm{Li} / 18 \mathrm{C}$ ), $\mathrm{Li} / 12 \mathrm{C}$ (and $\mathrm{Li} / 24 \mathrm{C}$ ), and $\mathrm{Li} / 36 \mathrm{C}$, respectively. The interlayer structures of stages 1 to 8 were also used for shifted k-point sampling from 24 to 2, respectively. The electron-occupation numbers were determined by the Gaussian smearing technique with a broadening parameter of $0.01 \mathrm{Ry}$. The atomic positions and cell parameters were optimized until the residual forces and stresses became less than $10^{-3} \mathrm{Ry} / \mathrm{bohr}(1 \mathrm{bohr}=0.52918 \AA)$ and $0.5 \mathrm{kbar}(1 \mathrm{bar}=105 \mathrm{~Pa})$, respectively. The average $\mathrm{C}-\mathrm{C}$ lengths multiplied by $\sqrt{3}$ and the average layer distances from the optimized cell parameters are plotted in Fig. S2. 
(a) AB stack

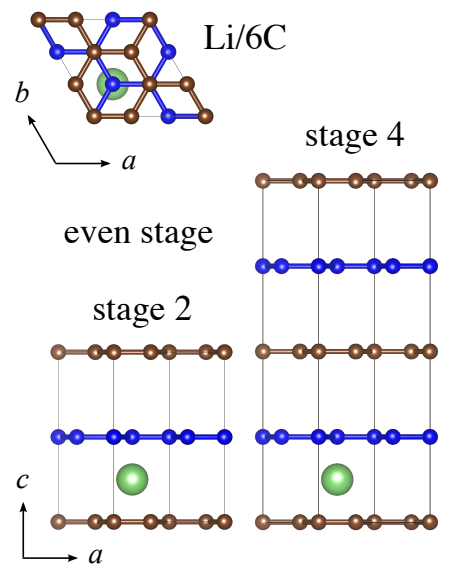

(b) mix stack
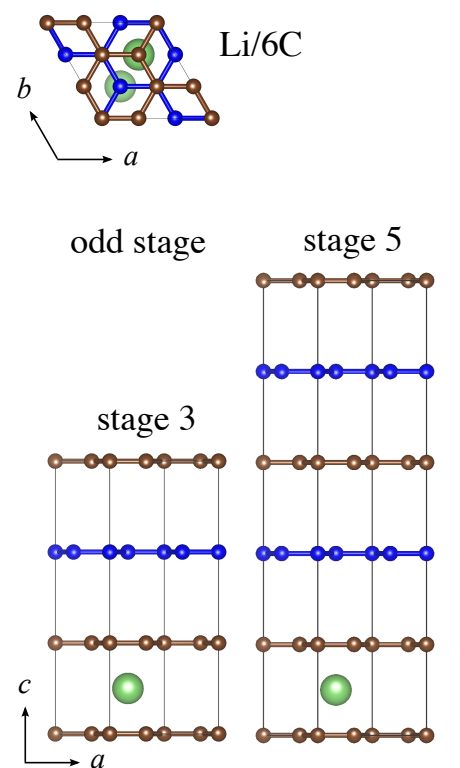

(d)

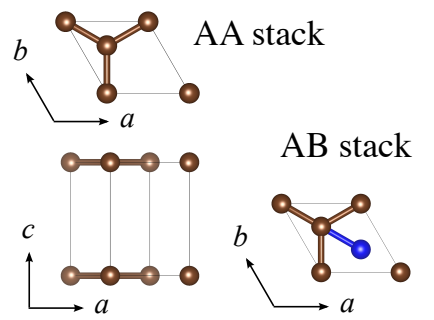

stage 3

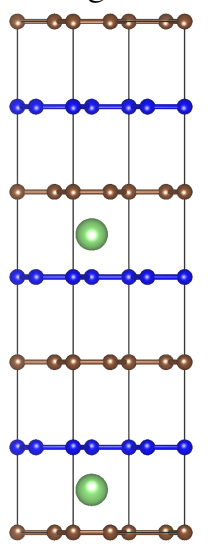

stage 4

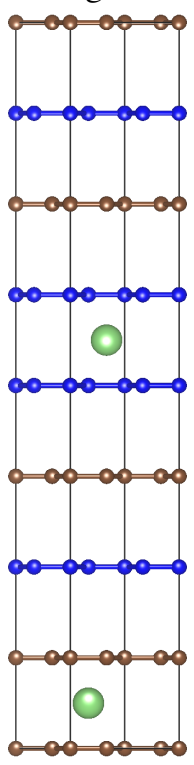

(c) $\operatorname{mix}-\mathrm{Li} / 9 \mathrm{C}-\mathrm{s} 2$
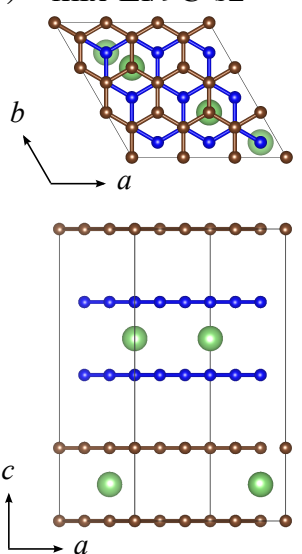

mix-Li/12C-s2

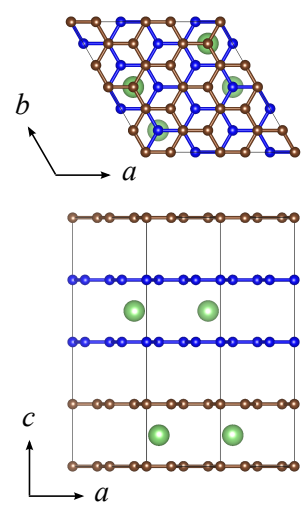

mix-Li/24C-s2
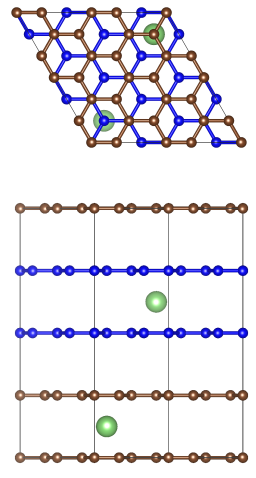

mix-Li/36C-s2

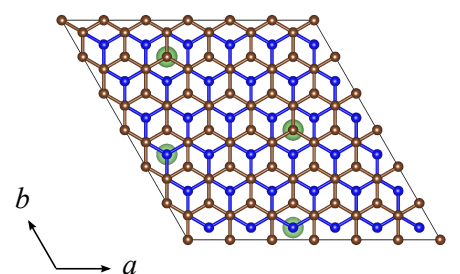

$\operatorname{mix}-\mathrm{Li} / 18 \mathrm{C}-\mathrm{s} 2$

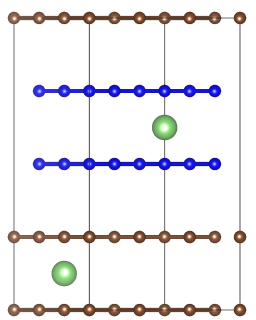

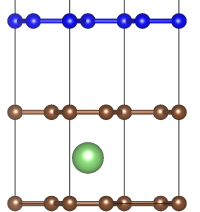

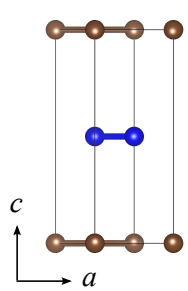

bcc Li

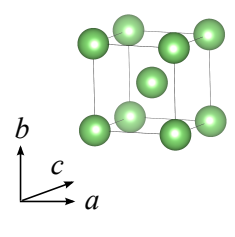

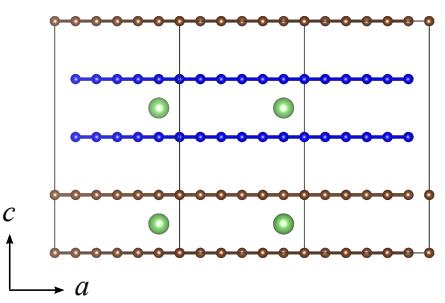

Figure S1. Crystal structures of (a) AB-Li/6C-sn ( $n=1,2,3$, and 4), (b) mix-Li/6C-s2-mix-Li/6C-s5, (c) mix-Li/9C-s2, mix-Li/12C-s2, mix-Li/18C-s2, mix-Li/24C-s2, and mix-Li/36C-s2, and (d) AA-C, AB-C, and bcc Li. The Li, C (in the A configuration), and C (in the B configuration) atoms are shown as green, brown, and blue spheres, respectively. The VESTA package was used to visualize the coordinates of the atoms. $^{\text {S9 }}$ 

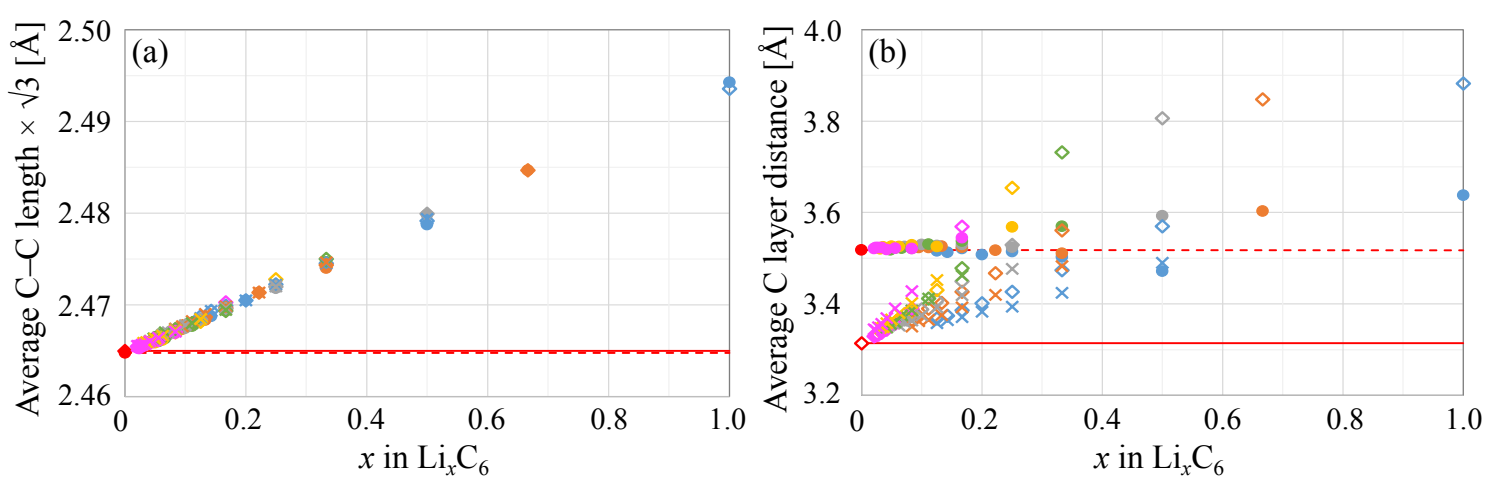

Figure S2. Average $\mathrm{C}-\mathrm{C}$ lengths multiplied by $\sqrt{3}$ and average $\mathrm{C}$ layer distances as a function of the $\mathrm{Li}$ composition. The blue, orange, gray, green, yellow, and magenta symbols represent the in-plane configurations of $\mathrm{Li} / 6 \mathrm{C}, \mathrm{Li} / 9 \mathrm{C}, \mathrm{Li} / 12 \mathrm{C}, \mathrm{Li} / 18 \mathrm{C}, \mathrm{Li} / 24 \mathrm{C}$, and $\mathrm{Li} / 36 \mathrm{C}$, respectively. The solid circles, open diamonds, and crosses represent the interlayer configurations of the $\mathrm{AA}, \mathrm{AB}$, and mixed stacks, respectively. The red solid circle and red diamond represent AA-C and AB-C, respectively. The red solid and dashed lines show the values of AA-C and AB-C, respectively, as guides for the eye. 


\section{S2. Computational details of the phonon calculations}

The phonon calculation scheme, which is implemented in $\mathrm{QE}$ using density functional perturbation theory, ${ }^{\mathrm{S} 10}$ was used with ultrasoft pseudopotentials. ${ }^{\mathrm{S} 3-\mathrm{S} 7}$ The vdW-DF2-B86R ${ }^{\mathrm{S} 8}$ and Perdew-BurkeErnzerhof ${ }^{\mathrm{S} 11}$ exchange-correlation functionals were used for the phonon calculations of $\mathrm{Li}_{x} \mathrm{C}_{6}$ and bcc $\mathrm{Li}$, respectively. The phonon frequencies (and eigenvectors) were obtained from the dynamical matrix on the q-point grids in reciprocal space and then interpolated to dense q-point grids by Fourier interpolation. The sampling of the k-point and q-point grids used for the phonon calculations is summarized in Table S1. The lattice parameters were taken from the DFT calculation results described in Section S1. The atomic positions were further relaxed until the residual forces became less than $10^{-4} \mathrm{Ry} / \mathrm{bohr}$. The other parameters for the electronic structure calculations were the same as those described in Section S1. The phonon densities of states (DOSs) were calculated for AB-C, AA-C, AA-Li/6C-s1-s3, mix-Li/6C-s3, AA-Li/9Cs1-s2, and bec Li (Fig. S4).

Table S1. Sampling of the k-point, q-point, and dense q-point grids used for the phonon calculations.

\begin{tabular}{cccc} 
Structure & k-point grid & q-point grid & dense q-point grid \\
\hline AB-C & $24 \times 24 \times 12$ & $12 \times 12 \times 6$ & $64 \times 64 \times 32$ \\
AA-C & $24 \times 24 \times 18$ & $12 \times 12 \times 9$ & $64 \times 64 \times 48$ \\
AA-Li/6C-s1 & $16 \times 16 \times 24$ & $8 \times 8 \times 12$ & $32 \times 32 \times 48$ \\
AA-Li/6C-s2 & $16 \times 16 \times 12$ & $8 \times 8 \times 6$ & $32 \times 32 \times 24$ \\
AA-Li/6C-s3 & $16 \times 16 \times 8$ & $8 \times 8 \times 4$ & $32 \times 32 \times 16$ \\
mix-Li/6C-s3 & $16 \times 16 \times 8$ & $8 \times 8 \times 4$ & $32 \times 32 \times 16$ \\
AA-Li/9C-s1 & $8 \times 8 \times 16$ & $4 \times 4 \times 8$ & $32 \times 32 \times 64$ \\
AA-Li/9C-s2 & $8 \times 8 \times 8$ & $4 \times 4 \times 4$ & $32 \times 32 \times 32$ \\
bcc Li & $16 \times 16 \times 16$ & $8 \times 8 \times 8$ & $64 \times 64 \times 64$
\end{tabular}



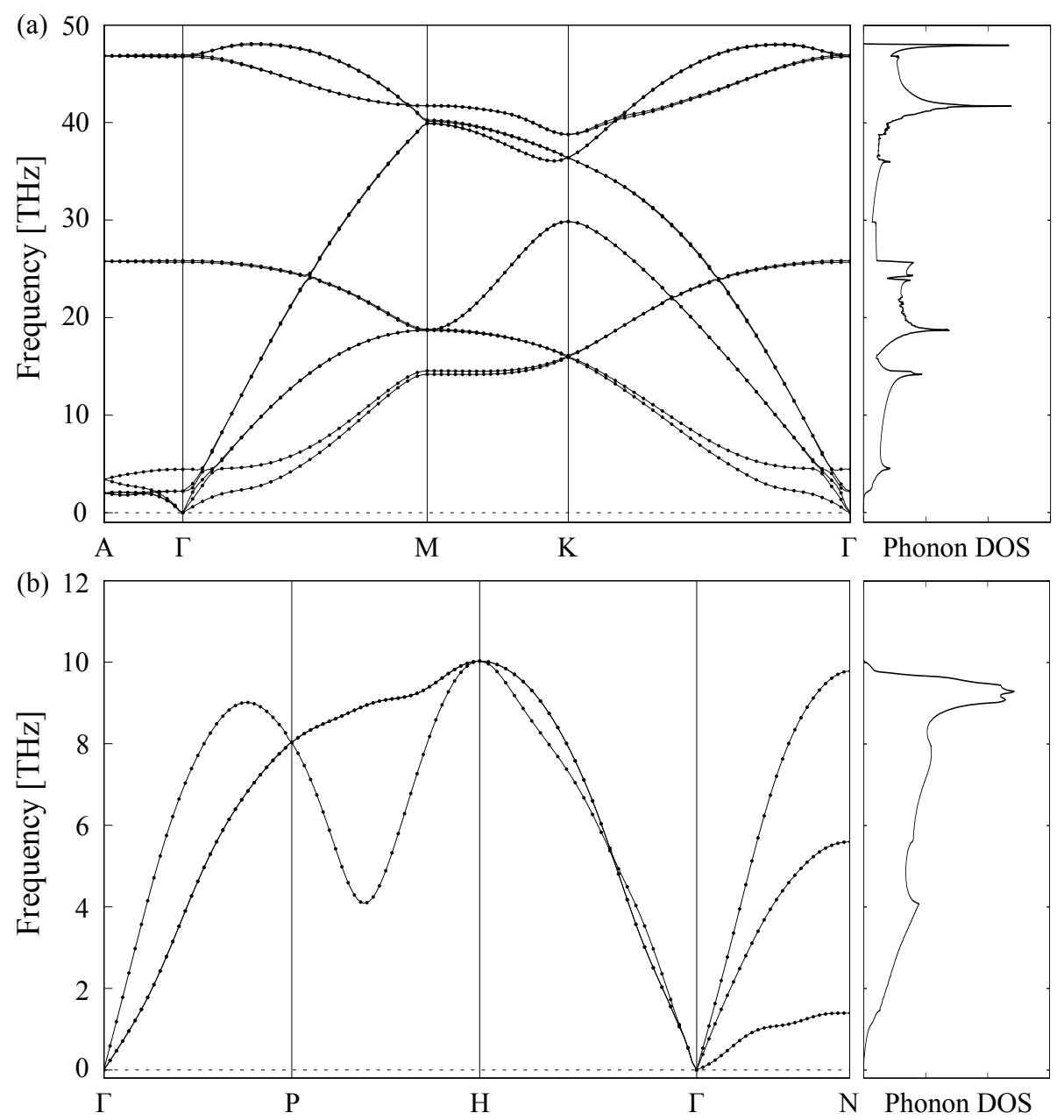

Figure S3. Phonon frequencies of (a) AB-C and (b) bcc Li passing through the high-symmetry q points in the Brillouin zones. The corresponding phonon DOSs are shown on the right-hand sides. 

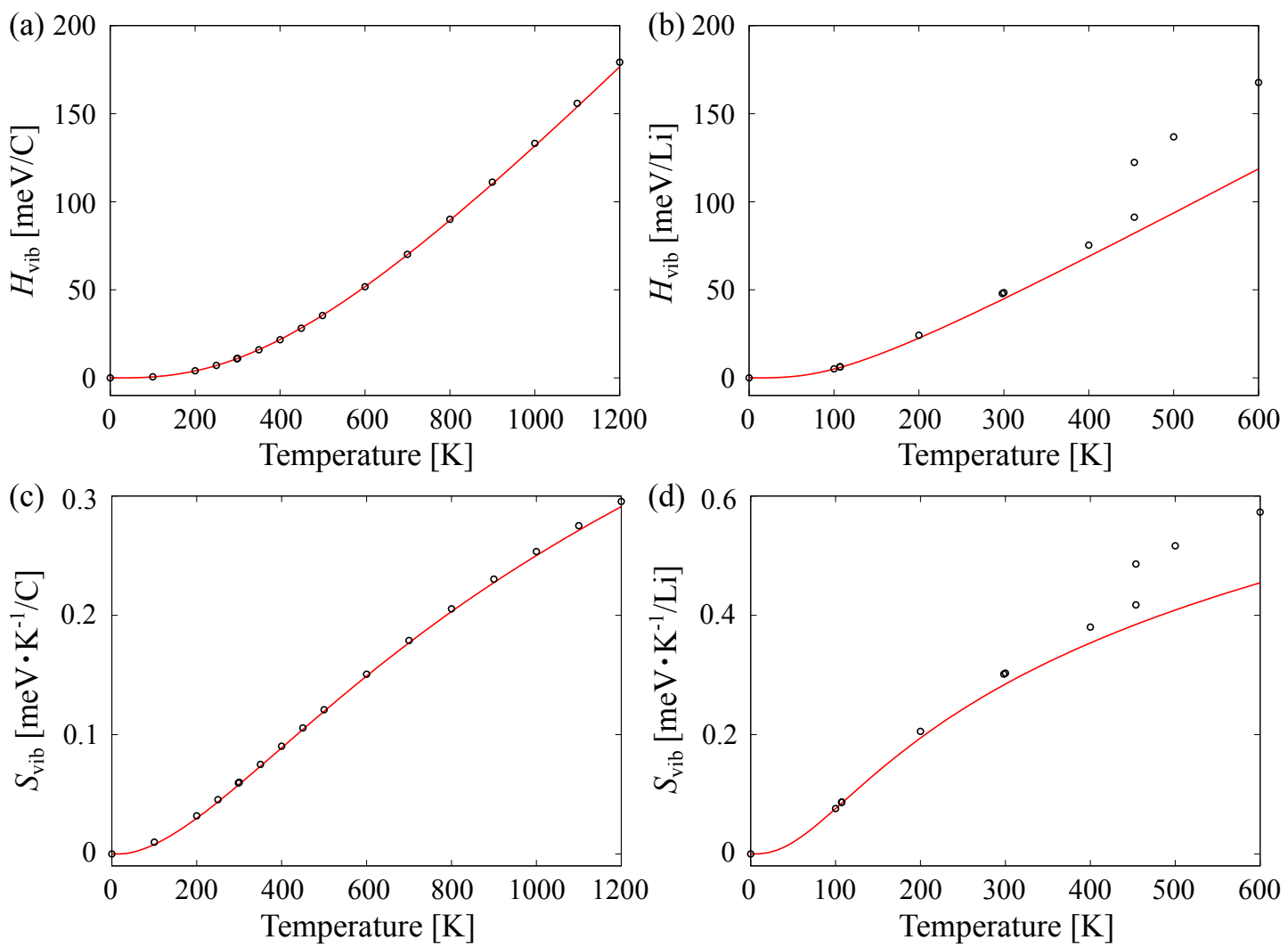

Figure S4. Vibrational enthalpies ( $H_{\mathrm{vib}}$, the values at $T=0$ are shifted to 0 ) of (a) AB-C and (b) bcc Li and vibrational entropies $\left(S_{\mathrm{vib}}\right)$ of (c) AB-C and (d) bcc Li as a function of temperature. The red lines are the values calculated by eqs. (5) and (6). The black open circles are the experimental values from refs. S12 and S13.

Table S2. Vibrational enthalpies $\left(H_{\mathrm{vib}}\right)$ and entropies $\left(S_{\mathrm{vib}}\right)$ of AB-C and bec Li at $T=300 \mathrm{~K}$.

$$
H_{\text {vib }}[\mathrm{kJ} / \mathrm{mol}] \quad S_{\text {vib }}[\mathrm{J} / \mathrm{mol} \cdot \mathrm{K}]
$$

\begin{tabular}{rcccc} 
Structure & Calculation & Experiment $^{\mathrm{S} 12}$ & Calculation & Experiment $^{\mathrm{S} 13}$ \\
\hline AB-C & 1.07 & 1.07 & 5.63 & 5.79 \\
bcc Li & 4.33 & 4.67 & 27.5 & 29.2
\end{tabular}


Table S3. Formation enthalpies $\left(\Delta H^{\mathrm{f}}\right)$ at $T=300 \mathrm{~K}$ and the corresponding structures, Li compositions, and stoichiometries on the convex hull in Fig. 3(a) and (b).

\begin{tabular}{cccc} 
Structure & $x$ in $\mathrm{Li}_{x} \mathrm{C}_{6}$ & Stoichiometry & $\Delta H^{\mathrm{f}}\left[\mathrm{meV}^{2} \mathrm{Li}_{x} \mathrm{C}_{6}\right]$ \\
\hline AB-C & 0 & $\mathrm{C}$ & 0 \\
mix-Li/18C-s6 & 0.0556 & $\mathrm{LiC}_{108}$ & -12.8 \\
mix-Li/18C-s4 & 0.0833 & $\mathrm{LiC}_{72}$ & -18.7 \\
mix-Li/12C-s5 & 0.100 & $\mathrm{LiC}_{60}$ & -22.2 \\
mix-Li/12C-s4 & 0.125 & $\mathrm{LiC}_{48}$ & -27.3 \\
AA-Li/6C-s2 & 0.500 & $\mathrm{LiC}_{12}$ & -94.6 \\
AA-Li/6C-s1 & 1 & $\mathrm{LiC}_{6}$ & -114.9
\end{tabular}

Table S4. Formation free energies $\left(\Delta G^{\mathrm{f}}\right)$ at $T=300 \mathrm{~K}$ and the corresponding structures, Li compositions, stoichiometries, and average $\mathrm{C}$ layer distances on the convex hull in Fig. 3(c) and (d).

\begin{tabular}{ccccc} 
Structure & $x$ in $\mathrm{Li}_{x} \mathrm{C}_{6}$ & Stoichiometry & $\Delta G^{\mathrm{f}}\left[\mathrm{meV} / \mathrm{Li}_{x} \mathrm{C}_{6}\right]$ & Layer distance $[\AA]$ \\
\hline AB-C & 0 & $\mathrm{C}$ & 0 & 3.314 \\
$\mathrm{AB}-\mathrm{Li} / 36 \mathrm{C}-\mathrm{s} 7$ & 0.0238 & $\mathrm{LiC}_{252}$ & -7.9 & 3.331 \\
$\mathrm{AB}-\mathrm{Li} / 36 \mathrm{C}-\mathrm{s} 5$ & 0.0333 & $\mathrm{LiC}_{180}$ & -10.5 & 3.337 \\
mix-Li/18C-s4 & 0.0833 & $\mathrm{LiC}_{72}$ & -22.8 & 3.386 \\
AA-Li/9C-s2 & 0.333 & $\mathrm{LiC}_{18}$ & -81.8 & 3.511 \\
AA-Li/6C-s2 & 0.500 & $\mathrm{LiC}_{12}$ & -115.3 & 3.472 \\
AA-Li/6C-s1 & 1 & $\mathrm{LiC}_{6}$ & -127.4 & 3.638
\end{tabular}



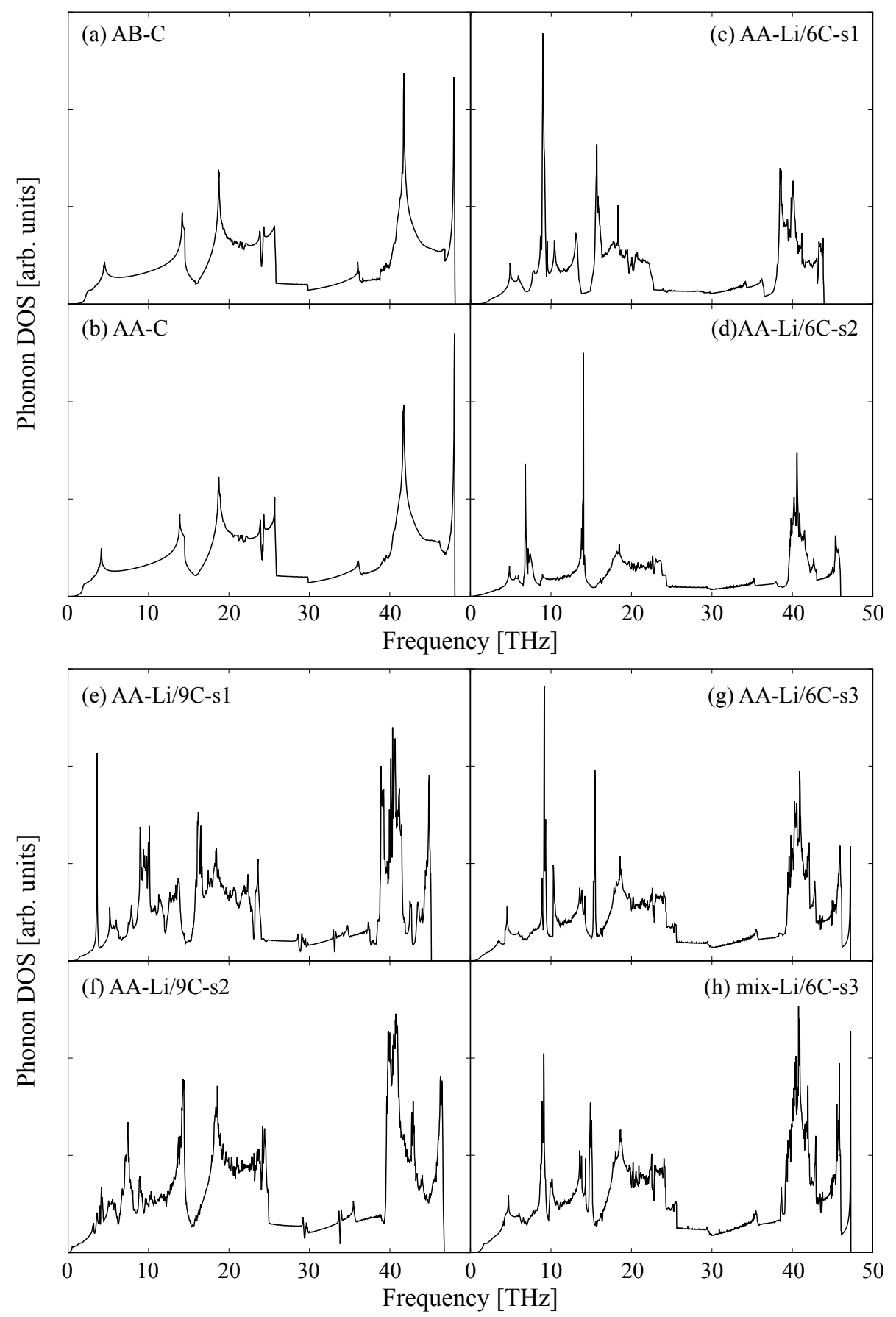

Figure S5. Phonon DOSs of (a) AB-C, (b) AA-C, (c) AA-Li/6C-s1, (d) AA-Li/6C-s2, (e) AA-Li/9C-s1, (f) AA-Li/9C-s2, (g) AA-Li/6C-s3, and (h) mix-Li/6C-s3. 


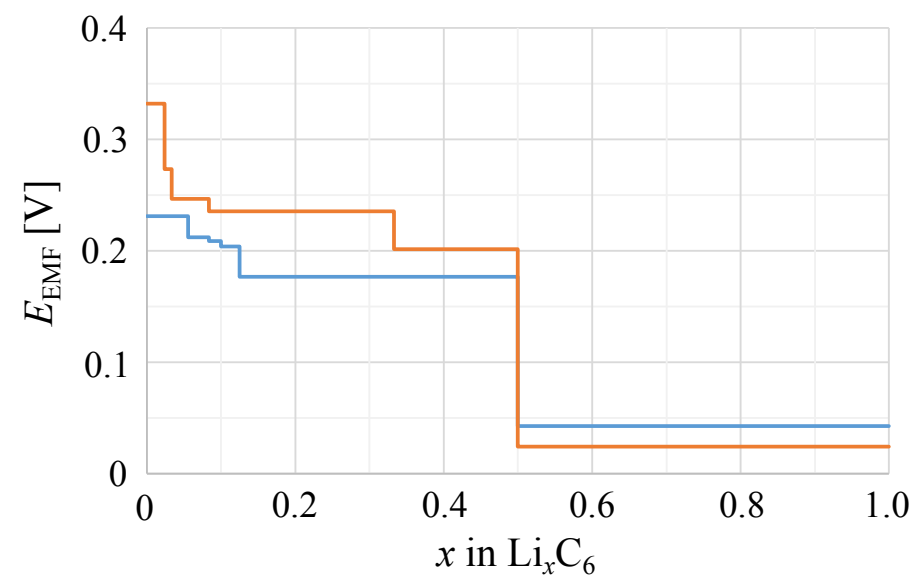

Figure S6. Electromotive force of $\mathrm{Li}_{x} \mathrm{C}_{6}$ as a function of the $\mathrm{Li}$ composition. The orange and blue lines represent $E_{\mathrm{EMF}}$ from the formation free energy and that approximated only by the enthalpy, respectively.

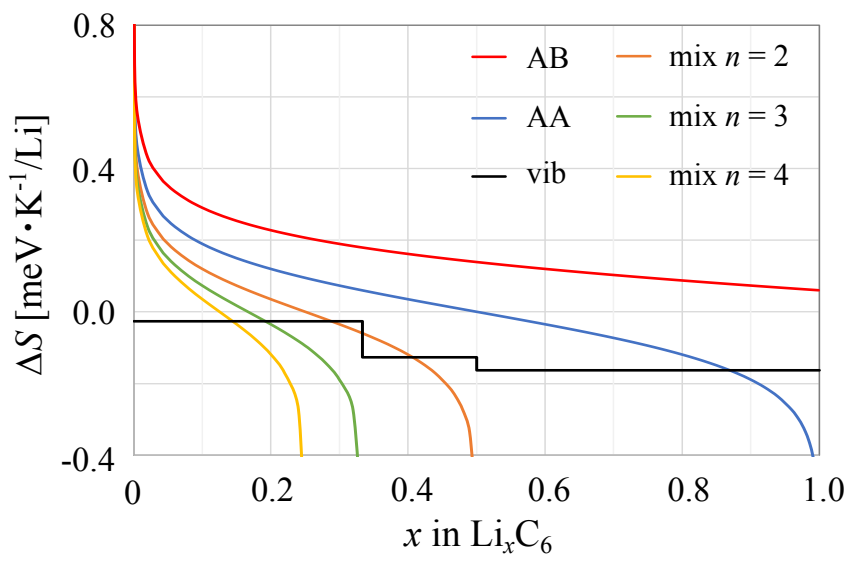

Figure S7. Components of the formation entropy $(\Delta S)$ in eqs. (15)-(17). The black line represents the vibrational part ( $\left.\Delta S_{\mathrm{vib}}\right)$. The red and blue lines represent the configurational parts ( $\left.\Delta S_{\text {conf }}\right)$ corresponding to the $\mathrm{AB}$ and $\mathrm{AA}$ stacks, respectively. The orange, green, and yellow lines represent $\Delta S_{\text {conf }}$ corresponding to the mixed stacks of stages 2,3 , and 4 , respectively. 


\section{REFERENCES}

(S1) Giannozzi, P.; Baroni, S.; Bonini, N.; Calandra, M.; Car, R.; Cavazzoni, C.; Ceresoli, D.; Chiarotti, G. L.; Cococcioni, M.; Dabo, I.; et al. QUANTUM ESPRESSO: A Modular and Open-Source Software Project for Quantum Simulations of Materials. J. Phys.: Condens. Matter 2009, 21, 395502.

(S2) Giannozzi, P.; Andreussi, O.; Brumme, T.; Bunau, O.; Nardelli M. B.; Calandra, M.; Car, R.; Cavazzoni, C.; Ceresoli, D.; Cococcioni, M.; et al. Advanced Capabilities for Materials Modeling with QUANTUM ESPRESSO. J. Phys.: Condens. Matter 2017, 29, 465901.

(S3) Vanderbilt, D. Soft Self-Consistent Pseudopotentials in a Generalized Eigenvalue Formalism. Phys. Rev. B 1990, 41, 7892-7895.

(S4) Rappe, A. M.; Rabe, K. M.; Kaxiras, E.; Joannopoulos, J. D. Optimized Pseudopotentials. Phys. Rev. $B$ 1990, 41, 1227-1230.

(S5) Dal Corso, A. Pseudopotentials periodic table: From H to Pu. Comput. Mater. Sci. 2014, 95, 337-350.

(S6) A Library of Ultrasoft and PAW Pseudopotentials. https://dalcorso.github.io/pslibrary/ (Accessed: November 25, 2021).

(S7) Louie, S. G.; Froyen, S.; Cohen, M. L. Nonlinear Ionic Pseudopotentials in Spin-Density-Functional Calculations. Phys. Rev. B 1982, 26, 1738-1742.

(S8) Hamada, I. van der Waals Density Functional Made Accurate. Phys. Rev. B 2014, 89, 121103(R).

(S9) Momma, K.; Izumi, F. VESTA: A Three-Dimensional Visualization System for Electronic and Structural Analysis. J. Appl. Crystallogr. 2011, 44, 1272-1276.

(S10) Baroni, S.; de Gironcoli, S.; Dal Corso, A. Phonons and Related Crystal Properties from DensityFunctional Perturbation Theory. Rev. Mod. Phys. 2001, 73, 515-562.

(S11) Perdew, J. P.; Burke, K.; Ernzerhof, M. Generalized Gradient Approximation Made Simple. Phys. Rev. Lett. 1996, 77, 3865-3868.

(S12) NIST-JANAF Thermochemical Tables. https://janaf.nist.gov/tables/C-002.html (accessed November 25, 2021).

(S13) NIST-JANAF Thermochemical Tables. https://janaf.nist.gov/tables/Li-001.html (accessed November 25, 2021). 